\title{
Dimerization of xanthene dyes in sol-gel titania films
}

Hiromasa Nishikiori, ${ }^{*},{ }^{\dagger}$ Rudi Agus Setiawan, ${ }^{\dagger}$ Sayaka Kawamoto ${ }^{\dagger}$ Shinya Takagi, ${ }^{\dagger}$ Katsuya Teshima, ${ }^{\dagger}$ Tsuneo Fujii ${ }^{\dagger \dagger}$

†Department of Environmental Science and Technology, Faculty of Engineering, Shinshu University, 4-17-1 Wakasato, Nagano 380-8553, Japan

${ }^{\dagger}$ Nagano Prefectural Institute of Technology, 813-8 Shimonogo, Ueda, Nagano 386-1211, Japan

Corresponding author: Hiromasa Nishikiori

Tel.: +81-26-269-5536

Fax: +81-26-269-5531

E-mail: nishiki@shinshu-u.ac.jp

Department of Environmental Science and Technology, Faculty of Engineering, Shinshu University, 4-17-1 Wakasato, Nagano 380-8553, Japan 


\section{Abstract}

Dye-dispersing titania gel films were prepared by the sol-gel method using the titanium alkoxide solutions containing the xanthene dyes; i.e., fluorescein, eosin Y, rhodamine 110, and rhodamine 123. Dimerization of the xanthene dyes in the films, depending on their concentrations, and their photoinduced electron transfer processes to the titania gels were investigated by spectroscopic and photoelectric techniques. The dye having a higher dimerization constant in solution formed a higher amount of the dimer in the titania gel. There was only a slight difference between the photoelectric conversion efficiencies of the monomer and dimer. The titanium species were coordinated to the carboxylate of the dyes. The dimers as well as the monomers were encapsulated in the narrow spaces of the gel films and strongly interacted with the surrounding titania matrix in the dye-dispersing titania gel films. The dye having a more nucleophilic carboxylate oxygen was easier to form the coordination complex with the titanium species. The dye-titania interaction played an important role in the injection from the dye to the titania conduction band.

Keywords: xanthene dyes; dimerization; photoelectric conversion; sol-gel reaction; titania 


\section{Introduction}

Laser dyes, such as xanthene dyes, are useful substances for utilizing photoenergy due to their high absorption and fluorescence efficiencies in the visible light regions. ${ }^{1}$ For the practical utilization of such photofunctional dyes, they are required to be incorporated in block or film-shaped materials. Fine nanocomposite films consisting of organic dye molecules and an inorganic matrix are expected to be incorporated in electronic devices such as photovoltaic cells. ${ }^{2-4}$ The sol-gel method enables the production of glasses and ceramics via hydrolysis and polycondensation reactions of metal alkoxides. ${ }^{5-7}$ The organic-inorganic hybridization is performed by mixing the organic compounds into the starting solution. The sol-gel coating is a useful way to easily provide such highly functional materials on the inactive plate surface..$^{5-9}$ The physicochemical properties of the systems should vary according to the progress of the sol-gel reaction. The variations are interesting and important for understanding the fundamental chemical reactions. The absorption and fluorescence spectral measurements determined the behavior of the organic molecules in the sol-gel reaction systems. ${ }^{10-15}$ The previous studies revealed the relationships between the physicochemical changes during the sol-gel-xerogel transitions and the spectroscopic properties of the organic dyes; i.e., rhodamine $\mathrm{B},{ }^{16,17}$ methylene blue, ${ }^{18}$ and 9 -aminoacridine, ${ }^{19}$ in the silicon alkoxide systems. It was reported that the dye molecules were highly dispersed in the sol-gel silica matrices and encapsulated in their small 
pores. $^{10-21}$

The sol-gel reaction using a titanium alkoxide sol containing the dye molecules also easily allows the formation of the dye-dispersing titania films, which are amorphous or nanocrystalline..$^{5-9}$ In such systems, the dye molecules can be highly dispersed on the surface of the individual titania nanoparticles without their aggregation. On the other hand, our previous study showed that rhodamine $\mathrm{B}(\mathrm{RhB})$ molecules strongly interact with $-\mathrm{TiOH}$ groups in the thin sol-gel films of the silica-titania, and some of them form a dimer around the $-\mathrm{TiOH}$ groups. ${ }^{22}$ Negishi et al. also reported that RhB molecules encapsulated in a bulk gel using the sol-gel reaction of the silicon and titanium alkoxides strongly interacted with the $-\mathrm{TiOH}$ group. ${ }^{23}$ The dye-titania interaction is very important for the electron injection process in the photovoltaic materials such as the dye-sensitized solar cells. ${ }^{2-4}$ The functional groups were induced in the sensitized dye molecules in order to form a strong bond between the dye chromophore and the titania surface. ${ }^{3,24,25}$ Each molecule prefers to strongly bond to the titania surface for an efficient electron injection and reducing the energy transfer between the molecules which form the aggregates.

In this study, the dye-dispersing titania gels were prepared from the dye-containing titanium alkoxide sols by a room temperature sol-gel process. We investigated the dimerization of the xanthene dyes in the films, depending on their concentrations, and their photoinduced electron transfer processes to the titania gels. Four xanthene dyes, 
fluorescein, eosin Y, rhodamine 110, and rhodamine 123, as shown in Scheme 1, were used as the sensitizers because they have high molar extinction coefficients for harvesting light energy $^{1}$ and a high solubility into ethanol used as the solvent of the sol-gel systems. The dye-titania interaction and photoinduced electron transfer can be evaluated by photocurrent measurements as previously reported. ${ }^{26-28}$ The spectroscopic and photoelectric conversion properties of the dye-dispersing titania electrodes were investigated in order to clarify the influences of the dye dispersion and aggregation during the electron transfer process.

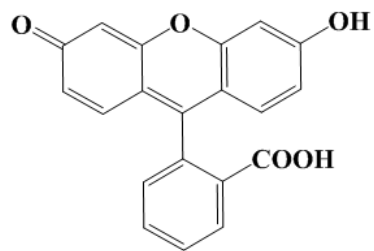

Fluorescein<smiles>Nc1ccc2c(-c3ccccc3C(=O)O)c3ccc(=[NH2+])cc-3oc2c1</smiles>

Rhodamine 110

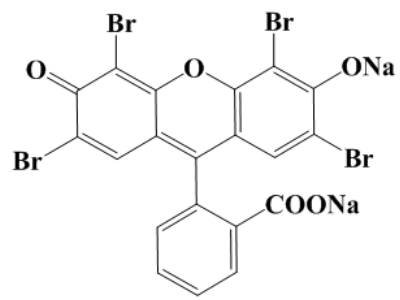

Eosin Y

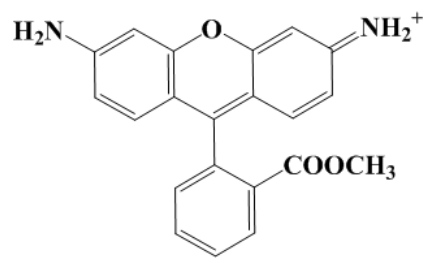

Rhodamine 123

Scheme 1. Molecular forms of xanthene dyes used in this study.

\section{Experimental}

\subsection{Materials}


Titanium tetraisopropoxide, ethanol, fluorescein, eosin Y, hydrochloric acid, nitric acid, diethylene glycol, iodine, lithium iodide, and sodium hydroxide (Wako Pure Chemical, S or reagent grade), and rhodamine 110 and rhodamine 123 (Kanto Chemical, reagent grade) were used without further purification. Water was ion-exchanged and distilled. Glass plates coated with the ITO transparent electrode (AGC Fabritech) were soaked in hydrochloric acid $\left(1.0 \mathrm{~mol} \mathrm{dm}^{-3}\right)$ for $2 \mathrm{~h}$ and then rinsed with water. The electrolyte used for the electrical measurements consisted of a diethylene glycol solution of iodine $\left(5.0 \times 10^{-2} \mathrm{~mol} \mathrm{dm}^{-3}\right)$ and lithium iodide $\left(0.50 \mathrm{~mol} \mathrm{dm}^{-3}\right)$.

\subsection{Preparation of electrodes}

The sol-gel reaction systems were prepared by mixing $5.0 \mathrm{~cm}^{3}$ of titanium tetraisopropoxide, $25.0 \mathrm{~cm}^{3}$ of ethanol, $0.21 \mathrm{~cm}^{3}$ of water, and nitric acid as the catalyst of the sol-gel reaction. The concentrations of nitric acid were $7.5 \times 10^{-2} \mathrm{~mol} \mathrm{dm}^{-3}$ in the systems. The xanthen dyes, fluorescein, eosin Y, rhodamine B, and rhodamine 110, were individually dissolved in the sol-gel reaction systems in which their concentrations were $1.0 \times 10^{-3}, 2.0 \times$ $10^{-3}, 3.0 \times 10^{-3}, 5.0 \times 10^{-3}$, and $1.0 \times 10^{-2} \mathrm{~mol} \mathrm{dm}^{-3}$. The dip-coated thin films were prepared from the systems in which the sol-gel reaction proceeded for 1 day to prepare the electrodes.

In order to prepare the electrode samples coated with the crystalline titania, the glass plates with the ITO transparent electrode were dip-coated in the dye-free system, and then heated at 
$500{ }^{\circ} \mathrm{C}$ for $30 \mathrm{~min}$. These electrodes were labeled E-0. Furthermore, the working electrodes were prepared in which the E-0 was dip-coated with each sol-gel system.

\subsection{Measurements}

The crystalline phase of the film samples was determined using an X-ray diffractometer (Rigaku RINT-2200V). The layer thickness of the electrode samples was estimated from their cross section using a field emission scanning electron microscope (Hitachi S-4100). The UV-visible absorption and fluorescence spectra of the prepared electrode samples were observed using a spectrophotometer (Shimadzu UV-3510) and a fluorescence spectrophotometer (Shimadzu RF-5300), respectively. The amounts of the dyes existing in the electrode samples were estimated from the absorption spectra of the dyes eluted by the 0.1 mol $\mathrm{dm}^{-3}$ hydrochloric acid or sodium hydroxide aqueous solution. The flakes of the dye and dye-dispersing film samples were pressed in $\mathrm{KBr}$ pellets and their IR spectra were obtained using an FTIR spectrophotometer (Shimadzu FTIR-8300).

The iodine-based electrolyte was allowed to soak into the space between the electrode sample and the counter Pt electrode. Monochromatic light obtained from the fluorescence spectrophotometer with a $150 \mathrm{~W}$ Xe short arc lamp (Ushio UXL-155) was irradiated on the electrodes for the spectroscopic measurements. Under light irradiation, the short circuit currents of the electrodes were measured using a digital multimeter (ADCMT 7461A). The intensity at each wavelength of the light source was obtained using a power meter (Molectron 
PM500A) in order to estimate the incident photon to current conversion efficiency (IPCE) and absorbed photon to current conversion efficiency (APCE) in the electrode samples. The light intensity was confirmed to correlate with the results of the potassium ferrioxalate actinometry. The visible absorbance of the present electrode samples was lower than 1.0 which was sufficient to measure the number of absorbed photons.

\section{Results and discussion}

\subsection{UV-vis absorption and photocurrent spectra of the dye-dispersing titania gel films}

No particle was observed in the SEM images of the dye-dispersing titania gel films regardless of the dye or dye concentration. No XRD peak was obtained in all the films. The gel films were concluded to be completely amorphous. The thickness of the dye-containing layer of the dye-dispersing titania gels was ca. $350 \mathrm{~nm}$ as previously reported. ${ }^{26-28}$

The concentration dependence of the UV-vis absorption and photocurrent spectra of the dye-dispersing titania gel films are shown in Figs. 1-4 for fluorescein, eosin Y, rhodamine 110, and rhodamine 123, respectively. The longitudinal axis of the photocurrent spectra indicates the IPCE value. The amounts of the dyes existing in the electrode samples were confirmed to be proportional to their initial concentrations in the sol-gel reaction systems. The dye amounts in all the films prepared from the sols containing $1.0 \times 10^{-2} \mathrm{~mol} \mathrm{dm}^{-3}$ of the 
dyes were ca. $5 \times 10^{-8}$ mol.

(a)

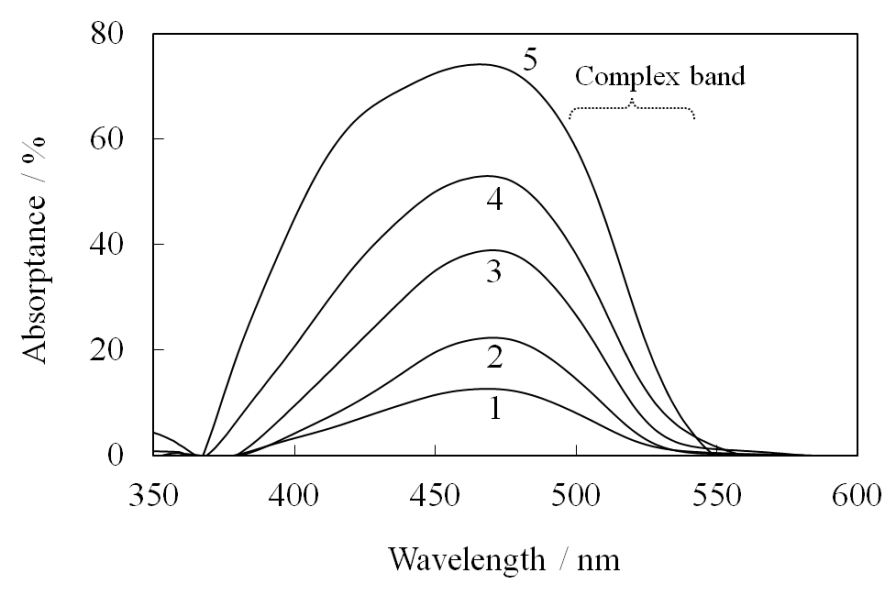

(b)

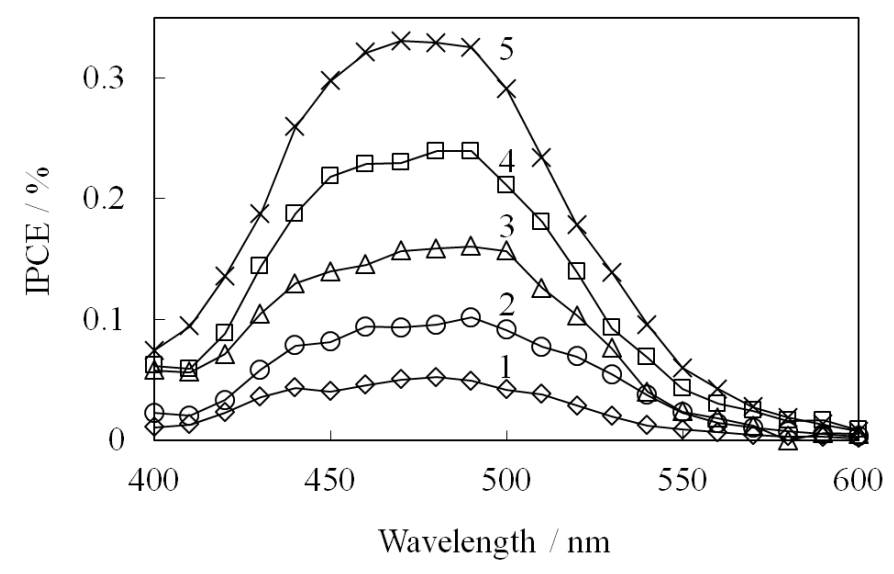

Figure 1. (a) UV-vis absorption and (b) IPCE spectra of the fluorescein-dispersing titania gel films prepared from the sols containing (1) $1.0 \times 10^{-3}$, (2) $2.0 \times 10^{-3}$, (3) $3.0 \times 10^{-3}$, (4) $5.0 \times 10^{-3}$, and (5) $1.0 \times 10^{-2} \mathrm{~mol} \mathrm{dm}^{-3}$ of fluorescein. 
(a)

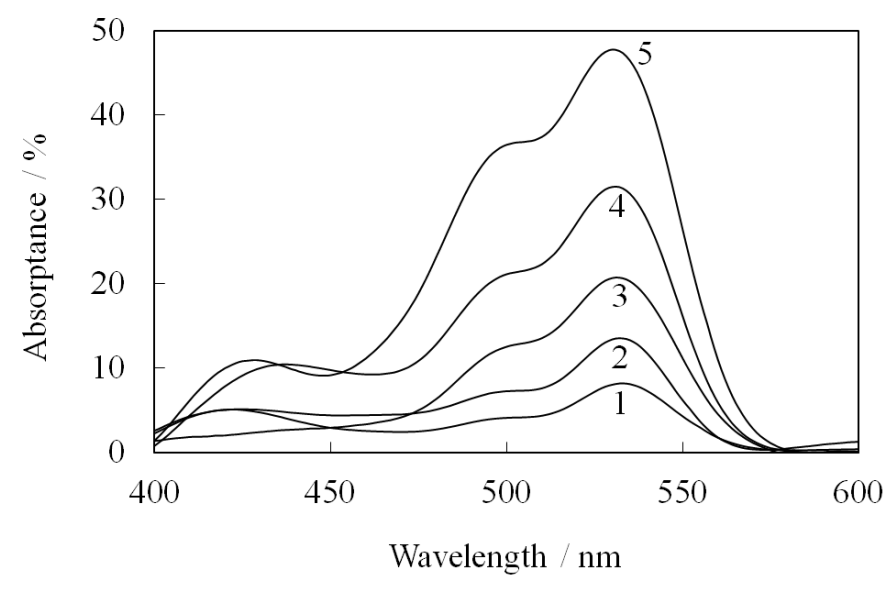

(b)

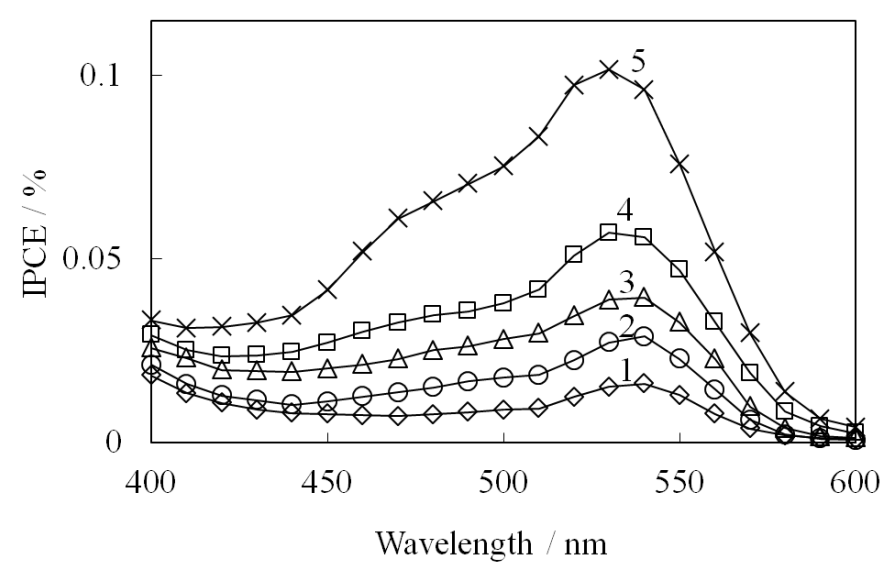

Figure 2. (a) UV-vis absorption and (b) IPCE spectra of the eosin Y-dispersing titania gel films prepared from the sols containing (1) $1.0 \times 10^{-3}$, (2) $2.0 \times 10^{-3}$, (3) $3.0 \times 10^{-3}$, (4) $5.0 \times$ $10^{-3}$, and (5) $1.0 \times 10^{-2} \mathrm{~mol} \mathrm{dm}^{-3}$ of eosin $\mathrm{Y}$. 
(a)

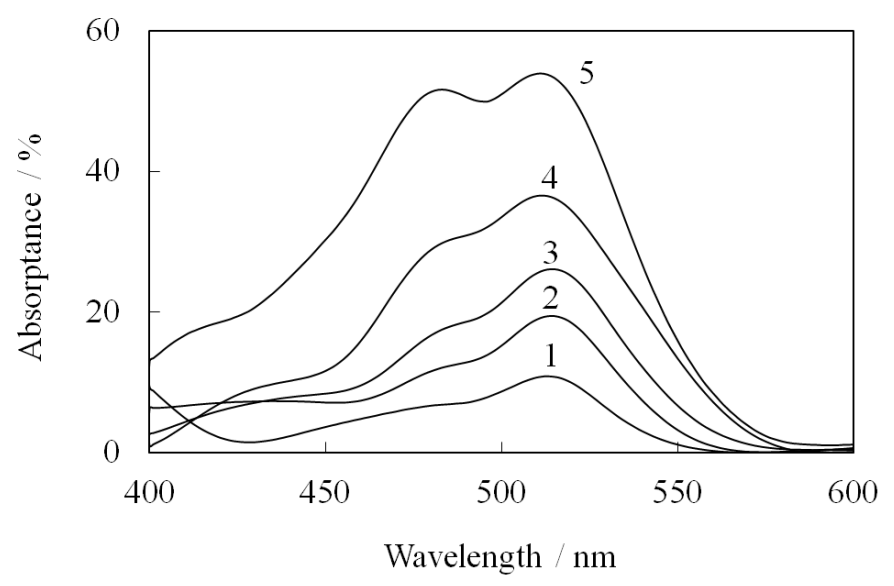

(b)

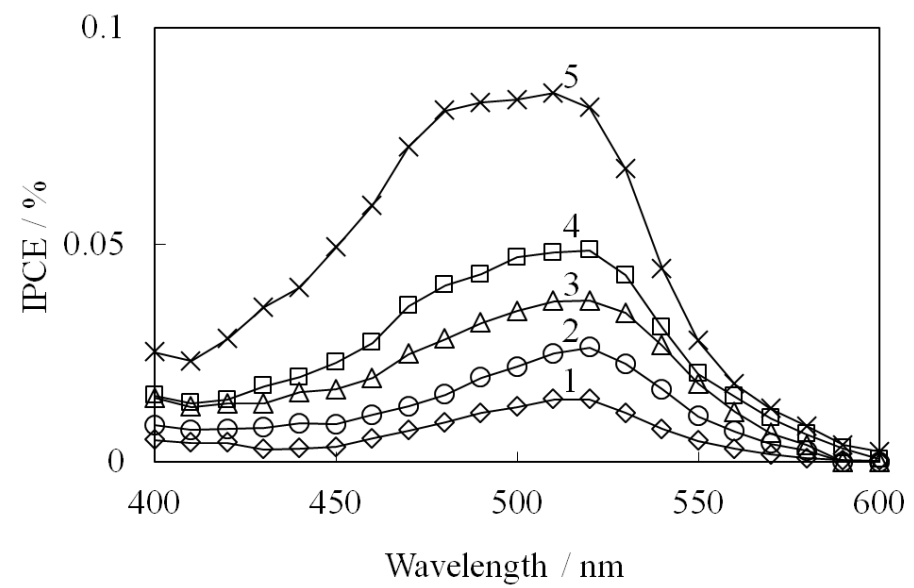

Figure 3. (a) UV-vis absorption and (b) IPCE spectra of the rhodamine 110-dispersing titania gel films prepared from the sols containing (1) $1.0 \times 10^{-3}$, (2) $2.0 \times 10^{-3}$, (3) $3.0 \times 10^{-3}$, (4) $5.0 \times 10^{-3}$, and (5) $1.0 \times 10^{-2} \mathrm{~mol} \mathrm{dm}^{-3}$ of rhodamine 110 . 
(a)

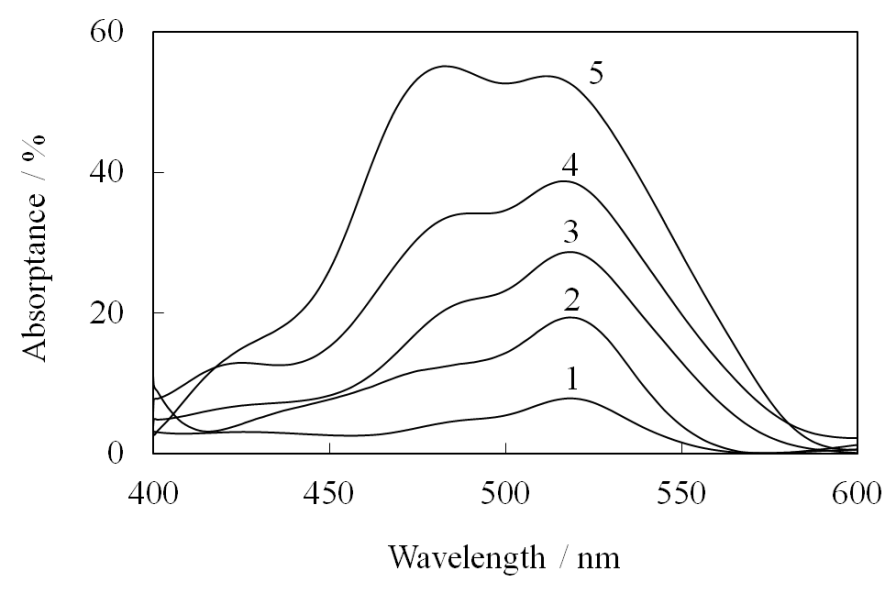

(b)

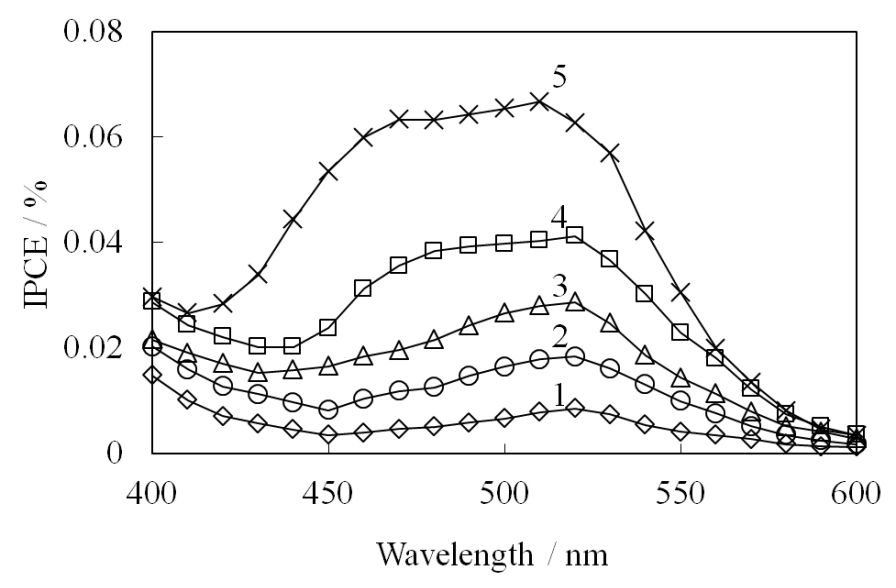

Figure 4. (a) UV-vis absorption and (b) IPCE spectra of the rhodamine 123-dispersing titania gel films prepared from the sols containing (1) $1.0 \times 10^{-3}$, (2) $2.0 \times 10^{-3}$, (3) $3.0 \times 10^{-3}$, (4) $5.0 \times 10^{-3}$, and (5) $1.0 \times 10^{-2} \mathrm{~mol} \mathrm{dm}^{-3}$ of rhodamine 123 .

The spectra of the fluorescein-dispersing titania gel films are located around $470 \mathrm{~nm}$, ranging over a wavelength wider than that observed in the solvents. The spectral peak position and shape only slightly depended on the dye content although the absorptance 
increased with an increase in the dye content. This result indicates that the main fluorescein species were the neutral or anion form (at 450-480 nm) and some fluorescein molecules existed as the dianion form (at around $490 \mathrm{~nm}$ ). ${ }^{29}$ In addition, the longer wavelength band (at 500-550 nm) indicated that a small number of fluorescein molecules formed the dianion-like species resulting from the strong interaction and chelating linkage between the carboxyl group of the dye and the titanium species, ${ }^{3,24,25,30,31}$ i.e., the polymers of titanium tetraisopropoxide or their aggregates. This is called the dye-titania complex in our study. The IPCE spectra of the fluorescein-dispersing electrodes had a peak at 480-490 $\mathrm{nm}$, which was located at a wavelength longer than that of the absorption spectra. This result indicated that the photocurrent more significantly resulted from the dye-titania complex than the other fluorescein species although the amount of the neutral and anion species was greater than that of the complex. The spectral peak and shape slightly changed with the dye content although the IPCE values increased with an increase in the dye content and the absorptance. The dianion species of the fluorescein forms its dimer in aqueous solutions. ${ }^{32,33}$ The neutral and anion species did not form the dimer in the titania gel films even though only a slight amount of the dianion species formed its dimer.

The eosin Y-dispersing titania gel with the lowest content of eosin Y exhibited an absorption peak at $530 \mathrm{~nm}$. The absorptance of this band increased with an increase in the dye content. The peak was located at a wavelength longer than that of the dianion species 
in aqueous solutions ${ }^{34}$ due to the ionic interaction with the titanium species. It is expected that the interaction of eosin $\mathrm{Y}$ with titania is weaker than that of fluorescein because the spectra of the eosin Y-dispersing titania gel films were sharper than those of the fluorescein-dispersing samples. In addition to the 530-nm band, the absorptance of the band at around $500 \mathrm{~nm}$ relatively increased with an increase in the dye content. This indicates the dimer formation of eosin $\mathrm{Y}$ in the film. ${ }^{32,35}$ The absorptance at the monomer peak was lower than that of the fluorescein-dispersing films due to the dimer formation. The IPCE spectra of the eosin Y-dispersing titania gel exhibited a peak at 530-540 nm similar to their absorption spectra. However, the spectra were broader in the longer wavelength region, indicating that the species interacting with the titanium species more efficiently generated the electricity. The IPCE values due to the dimer seem to increase with an increase in the dye content.

Rhodamine 110 and rhodamine 123 exhibit very similar absorption spectra because the difference in their structure is only the carboxyl or methyl carboxylate group. The absorption peaks of the rhodamine 110-dispersing and rhodamine 123-dispersing titania gels with their lowest contents are observed at 513 and $518 \mathrm{~nm}$, respectively. The cation monomers of rhodamines 110 and 123 are reported to exhibit an absorption peak at 499 and $500 \mathrm{~nm}$ in neutral aqueous solutions, respectively. ${ }^{36}$ The absorptance of these bands increased with an increase in the dye content. The spectra were broader than those of their 
cation species in aqueous solutions due to the ionic interaction with the titanium species. In addition to these absorption bands, the dimer bands at around $480 \mathrm{~nm}$ relatively increased with an increase in the dye content. ${ }^{37}$ The absorptance values at the monomer peaks were lower than that of the fluorescein-dispersing films due to the dimer formation. The dimer band intensity of the rhodamine 123 sample was somewhat stronger than that of the rhodamine 110 sample. These dimers should be the nonfluorescent $\mathrm{H}$-dimers because the bands mainly appeared at a wavelength shorter than the monomer bands. ${ }^{17,38,39}$ Actually, only a slight fluorescence was observed in the present systems due to the effective fluorescence quenching by the titania gel even though a low amount of the fluorescent J-dimers were formed as shown in the longer wavelength region than the monomer bands. The IPCE spectra of the rhodamine 110-dispersing and rhodamine 123-dispersing titania electrodes exhibited a peak at 510-520 nm similar to their absorption spectra. The IPCE values due to the dimers seem to increase with an increase in the dye content.

\subsection{Influence of dimer formation on the photoinduced electron injection from the dye to}

\section{the titania}

Fig. 5 shows the ratio of the absorbance for the dimer peak to that for the monomer peak observed in the dye-dispersing titania gel films versus the dye content. The result for the fluorescein-dispersing samples was left out from this figure because it did not form the dimer.

The slope of this graph indicates the dimerization tendency of the dye. The order of the 
slope corresponded to that of the dimerization constant in aqueous solutions, i.e., those of fluorescein, eosin $\mathrm{Y}$, rhodamine 110, and rhodamine 123 are 5, 110, 1000, and $2800 \mathrm{~mol}^{-1}$ $\mathrm{dm}^{3}$, respectively. ${ }^{32,33}$ The dimerization constants, $K_{\mathrm{d}}=[$ Dimer $] /[\text { Monomer }]^{2}$, of rhodamine 110 and rhodamine 123 were obtained from the dependence of their absorption spectra on the dye concentrations in this study. It was reported that the dimerization constants of the esterified rhodamines are higher than those of the unesterified rhodamines. ${ }^{37}$ The xanthene dyes usually form the dimer in aqueous solutions. ${ }^{32,33,35}$ In our previous study, the monomer of the dyes was the preferential species in the present sol-gel reaction systems containing a small amount of water until the gelation occurred. The dye molecules formed the dimer just after preparing the dip-coated thin film from the sol-gel system during increasing in the concentration of water with evaporation of alcohol. ${ }^{16-19,22}$ The concentrations of the dyes in the dye-dispersing titania films were estimated to be $1-3 \times 10^{-1}$ mol $\mathrm{dm}^{-3}$ from their absorbance and molar extinction coefficient and the film thickness. The dye molecules can reasonably form the dimers in the films because the average intermolecular distance is estimated to be $2-3 \mathrm{~nm}$. 


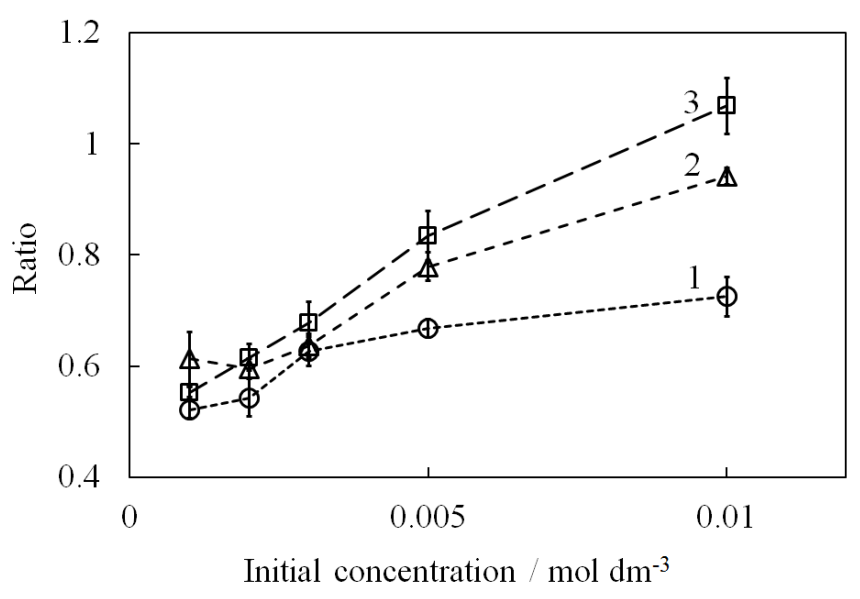

Figure 5. Ratio of the absorbance for the dimer peak to that for the monomer peak observed in the (1) eosin Y-, (2) rhodamine 110-, and (3) rhodamine 123-dispersing titania gel films versus their initial concentrations in the sols.

Fig. 6 shows the APCE values for the monomer and dimer peaks of the dye-dispersing titania gel films versus the dye content. The APCE value did not depend on the dye content in all the dye-dispersing titania gels. The APCE value at the dimer peak was similar to that at the monomer peak, indicating that the electron injection from the dye to the titania was slightly affected by the dimer formation. The order of the APCE value of the dye-dispersing titania gel films indicated that of the strong interaction between the dye and titanium species related to the electron injection efficiency. The proton dissociation constants, $\mathrm{p} K_{\mathrm{a}}$ values, of the carboxyl groups of fluorescein, eosin $\mathrm{Y}$, and rhodamine 110 are $4.45,3.75$, and 3.0, respectively. ${ }^{34,36}$ The carboxylate oxygen of fluorescein is more nucleophilic than that of the others and more easily forms the coordination complex with 
metal species. Additionally, the quinone-like carbonyl group of the xanthene ring in fluorescein and eosin Y should also interact with the titanium species. ${ }^{26}$ This type of complex formation strongly depends on $\mathrm{p} K_{\mathrm{a}}$ values of the hydroxyl group of the xanthene ring, which are 6.80 for fluorescein and 2.81 for eosin $\mathrm{Y}^{34}$ We previously reported that fluorescein more strongly bonded to the titanium species than eosin $\mathrm{Y}^{26}$ Based on the IR analysis, the significant shifts in the carboxylate and hydroxyl bands were observed due to the interaction between the functional groups and the titanium species of titania. ${ }^{26}$ On the other hand, the IR peaks of rhodamine 110 and rhodamine 123 were not significantly shifted by their dispersion into the titania gel although the carboxyl $\mathrm{C}=\mathrm{O}$ peak of rhodamine 110 at 1710 $\mathrm{cm}^{-1}$ disappeared due to its transformation to the carboxylate. Rhodamines more weakly interact with the titanium species than fluorescein and eosin $\mathrm{Y}$ having a hydroxyl group on the xanthene ring. Rhodamine 123 is expected to weakly interact with the titanium species compared to rhodamine 110 due to having a methyl carboxylate group. Therefore, the APCE value of the rhodamine 123 sample was lower than that of the rhodamine 110 sample. The high efficiency was due to the effect of the anchoring groups.

These efficiency values were much lower than those of the general dye-sensitized solar cells because the very thin amorphous titania films were used for the semiconductor layers in the present electrodes in order to obtain their exact absorptance and APCE values. In a previous study, the steam treatment enhanced the photoelectric conversion efficiency of the 
fluorescein-dispersing titania gel due to not only crystallization of the titania gel, but also the dye-titania surface complex formation. ${ }^{26-28}$ Furthermore, the IPCE value at $500 \mathrm{~nm}, 17 \%$, and internal energy conversion efficiency, $1.1 \%$, were obtained using a thicker titania electrode coated with the fluorescein-dispersing titania film. ${ }^{40}$ 
(a)

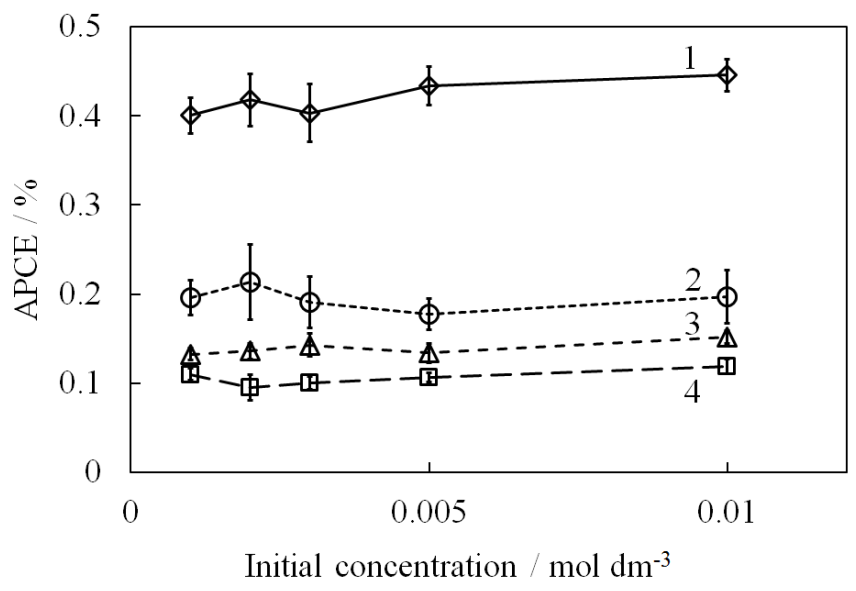

(b)

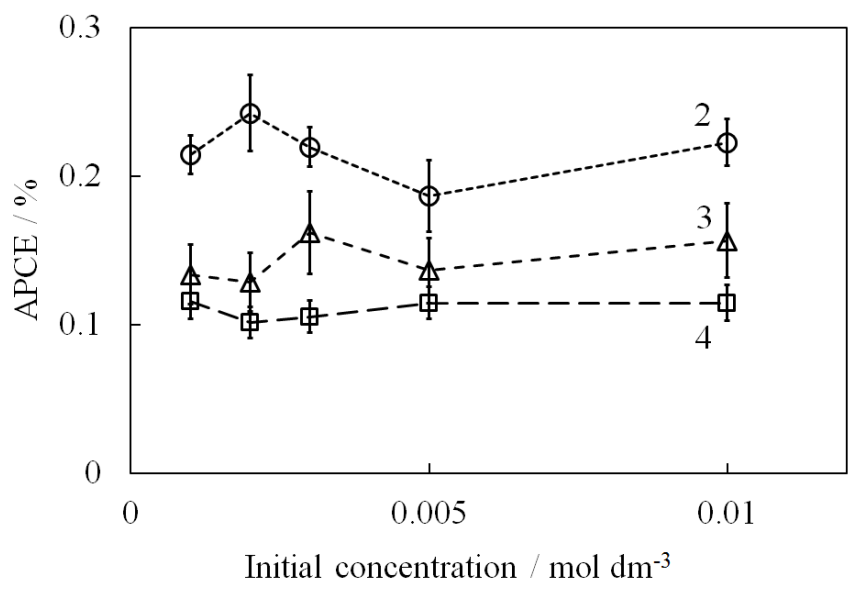

Figure 6. APCE values for the (a) monomer and (b) dimer peaks of the (1) fluorescein-, (2) eosin Y-, (3) rhodamine 110-, and (4) rhodamine 123-dispersing titania gel films versus their initial concentrations in the sols.

The APCE value depends on the LUMO level of the dye, which is an important factor for the rate of electron injection into the titania conduction band. The LUMO levels of such xanthene dyes are reported to be not significantly different, i.e., $-0.58--0.55$ vs. NHE 
(normal hydrogen electrode). ${ }^{41,42}$ The LUMO levels of the present xanthene dyes can be more negative than the level of the titania conduction band edge (ca. -0.5 vs. NHE). ${ }^{43}$ However, an efficient electron injection requires $0.2 \mathrm{eV}$ of driving force. ${ }^{44}$ In a previous study, the dye-titania complex formation caused the negative shift in the conduction band potential by ca. $0.2 \mathrm{~V}$ due to an increase in the negative charge density on the titania surface. ${ }^{26}$ Therefore, the dye-titania interaction is expected to be more effective for the electron injection rather than the small difference in the original LUMO levels of the xanthene dyes.

Fig. 7 shows the ratio of the APCE value for the dimer peak to that for the monomer peak versus the dye content. The results indicated that the efficiencies for the monomer and dimer were almost equal regardless of the dyes and their contents. The electrons in the dimers were injected into the titania conduction band in the same way as those in the monomers. The dye aggregation on the titania surface is a serious problem of the conventional dye-sensitized solar cells because it suppresses the electron injection. ${ }^{45-47}$ The dimers were encapsulated in the narrow spaces in the gel films and strongly interacted with the surrounding titania matrix in the dye-dispersing titania gel films, which were different from the conventional dye-adsorbing titania systems. It is presumed that the electron injection directly occurred from the chromophore of the two xanthene rings in the dimer to the titania without going through the benzene ring. 


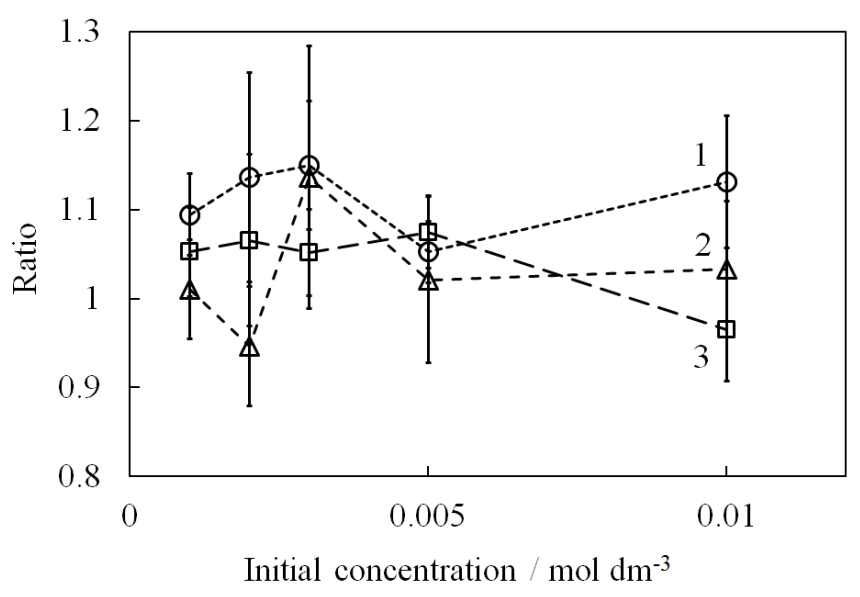

Figure 7. Ratio of the APCE value for the dimer peak to that for the monomer peak observed in the (1) eosin Y-, (2) rhodamine 110-, and (3) rhodamine 123-dispersing titania gel films versus their initial concentrations in the sols.

\section{Conclusions}

The dye-dispersing titania gels were prepared from the dye-containing titanium alkoxide sols by a room temperature sol-gel process. Dimerization of the xanthene dyes in the films, depending on their concentrations, and their photoinduced electron transfer processes to the titania gels were investigated using four xanthene dyes, fluorescein, eosin Y, rhodamine 110, and rhodamine 123, as the sensitizers. The spectroscopic and photoelectric conversion properties of the dye-dispersing titania electrodes were examined in order to clarify the influences of the dye dispersion and aggregation on the electron transfer process. The APCE values for the dye-dispersing titania electrodes depended on the extent of the interaction between the dye and titanium species related to the electron injection efficiency. 
The dye having a higher dimerization constant in solution formed a higher amount of the dimer in the titania gel. There were only a slight difference between the photoelectric conversion efficiencies of the monomer and dimer. The titanium species were coordinated to the carboxylate of the dyes. The dye having a more nucleophilic carboxylate oxygen more easily formed the coordination complex with the titanium species. The dye-titania interaction played an important role in the injection from the dye to the titania conduction band. The dye aggregation on the titania surface is a serious problem of the conventional dye-sensitized solar cells because it suppresses the electron injection. However, in the present systems, the electrons in the dimers were injected into the titania conduction band in the same way as those in the monomers. The dimers as well as the monomers were encapsulated in the narrow spaces of the gel films and strongly interacted with the surrounding titania matrix in the dye-dispersing titania gel films.

\section{Acknowledgment}

This work was supported by JSPS KAKENHI Grant Number 24550153.

\section{References}

1 M. Maeda, Laser Dyes: Properties of Organic Compounds for Dye Lasers; Academic Press: Tokyo, Japan, 1984. 
2 M. Grätzel, J. Photochem. Photobiol., C, 2003, 4, 145-153.

3 D. El Mekkawi and M. S. A. Abdel-Mottaleb, Int. J. Photoenergy, 2005, 7, 95-101.

4 G. D. Sharma, P. Balraju, M. Kumar and M. S. Roy, Mater. Sci. Eng. B, 2009, 162, $32-39$.

5 H. Dislich, Angew. Chem., Int. Ed. Engl., 1971, 10, 363-370.

6 H. Dislich, J. Non-Cryst. Solids, 1983, 57, 371-388.

7 C. J. Brinker and G. W. Scherer, Sol-Gel Science: The Physics and Chemistry of Sol-Gel Processing, Academic Press, San Diego, 1990.

8 C. J. Brinker, G. C. Frye, A. J. Hurd and C. S. Ashley, Thin Solid Films, 1991, 201, 97-108.

9 C. J. Brinker, A. J. Hurd, G. C. Frye, P. R. Schunk and C. S. Ashley, J. Ceram. Soc. Jpn., $1991,99,862-877$.

10 D. Avnir, D. Levy and R. Reisfeld, J. Phys. Chem., 1984, 88, 5956-5959.

11 R. Reisfeld, R. Zusman, Y. Cohen and M. Eyal, Chem. Phys. Lett. 1988, 147, 142-147.

12 T. Fujii, A. Ishii and M. Anpo, J. Photochem. Photobiol., A, 1990, 54, 231-237.

13 U. Narang, F. V. Bright and P. N. Prasad, Appl. Spectrosc., 1993, 47, 229, 229-234.

14 F. del Monte and D. Levy, Chem. Mater., 1995, 7, 292-298.

15 P. H. Chang, H. C. Tsai, Y. R. Chen, J. Y. Chen and G. H. Hsiue, Langmuir, 2008, 24, $11921-11927$. 
16 T. Fujii, H. Nishikiori and T. Tamura, Chem. Phys. Lett., 1995, 233, 424-429.

17 H. Nishikiori and T. Fujii, J. Phys. Chem. B, 1997, 101, 3680-3687.

18 H. Nishikiori, S. Nagaya, N. Tanaka, A. Katsuki and T. Fujii, Bull. Chem. Soc. Jpn., 1999, 72, 915-921.

19 H. Nishikiori, N. Tanaka, Y. Minami, A. Katsuki and T. Fujii, J. Photochem. Photobiol., A, 2010, 212, 62-67.

20 F. del Monte, M. L. Ferrer and D. Levy, Langmuir, 2001, 17, 4812-4817.

21 F. del Monte, M. L. Ferrer and D. Levy, J. Mater. Chem., 2001, 11, 1745-1751.

22 H. Nishikiori, N. Tanaka and T. Fujii, J. Sol-Gel Sci. Technol., 2001, 20, 95-104.

23 N. Negishi, M. Fujino, H. Yamashita, M. A. Fox and M. Anpo, Langmuir, 1994, 10, $1772-1776$.

24 M. Hilgendorff and V. Sundström, J. Phys. Chem. B, 1998, 102, 10505-10514.

25 G. Ramakrishna and H. N. Ghosh, J. Phys. Chem. B, 2001, 105, 7000-7008.

26 H. Nishikiori, Y. Uesugi, S. Takami, R. A. Setiawan, T. Fujii, W. Qian and M. A. El-Sayed, J. Phys. Chem. C, 2011, 115, 2880-2887.

27 H. Nishikiori, Y. Uesugi, R. A. Setiawan, T. Fujii, W. Qian and M. A. El-Sayed, J. Phys. Chem. C, 2012, 116, 4848-4854.

28 H. Nishikiori, R. A. Setiawan, K. Miyamoto, G. Sukmono, Y. Uesugi, K. Teshima and T. Fujii, RSC Adv., 2012, 2, 4258-4267. 
29 T. Fujii, A. Ishii, N. Takusagawa and M. Anpo, Res. Chem. Intermed., 1992, 17, 1-14.

30 G. Benkö, M. Hilgendorff, A. P. Yartsev and V. Sundström, J. Phys. Chem. B, 2001, 105, 967-974.

31 J. N. O'Shea, J. B. Taylor and E. F. Smith, Surf. Sci., 2004, 548, 317-323.

32 K. K. Rohatgi and A. K. Mukhopadhyay, J. Phys. Chem., 1972, 76, 3970-3973.

33 I. López Arbeloa, J. Chem. Soc., Faraday Trans. 2, 1981, 77, 1725-1733.

34 N. O. Mchedlov-Petrossyan and V. N. Kleshchevnikova, J. Chem. Soc. Faraday Trans., 1994, 90, 629-640.

35 I. López Arbeloa, Dyes Pigments, 1983, 4, 211-220.

36 M. El Baraka, M. Deumié and P. Viallet, J. Photochem. Photobiol., A, 1991, 62, $195-216$.

37 M. M. Wong and Z. A. Schelly, J. Phys. Chem. 1974, 78, 1891-1895.

38 F. del Monte and D. Levy, J. Phys. Chem. B, 1999, 103, 8080-8086.

39 F. del Monte, J. D. Mackenzie and D. Levy, Langmuir, 2000, 16, 7377-7382.

40 H. Nishikiori, Y. Uesugi, R. A. Setiawan and K. Teshima, J. Soc. Inorg. Mater. Jpn., 2012, 19, 237-242.

41 M. S. Chan and J. R. Bolton, Solar Energy 1980, 24, 561-574.

42 J. Yu and T. Zhou, J. Electroanal. Chem., 2001, 504, 89-95.

43 A. Hagfeldt and M. Grätzel, Chem. Rev., 1995, 95, 49-68. 
44 K. Hara, T. Sato, R. Katoh, A. Furube, Y. Ohga, A. Shinpo, S. Suga, K. Sayama, H. Sugihara and H. Arakawa, J. Phys. Chem. B, 2003, 107, 4693-4700.

45 A. C. Khazraji, S. Hotchandani, S. Das and P. V. Kamat, J. Phys. Chem. B, 1999, 103, $4693-4700$.

46 K. Keis, J. Lindgren, S. Lindquist and A. Hagfeldt, Langmuir, 2000, 16, 4688-4694.

47 B. Wenger, M. Grätzel and J. Moser, J. Am. Chem. Soc., 2005, 127, 12150-12151. 